\title{
L'apprentissage hybride en sciences de gestion : ce qu'en pensent les étudiants
}

Jean-Pierre Béchard, Catherine Carré, Gabriella Frankel, Zoffirath Dissou, Céline Bazinet, Gilles Cazabon et Yves Goyette

\section{OpenEdition}

Édition électronique

URL : http://journals.openedition.org/ripes/784

DOI : 10.4000/ripes.784

ISSN : 2076-8427

Éditeur

Association internationale de pédagogie universitaire

Référence électronique

Jean-Pierre Béchard, Catherine Carré, Gabriella Frankel, Zoffirath Dissou, Céline Bazinet, Gilles Cazabon et Yves Goyette, «L'apprentissage hybride en sciences de gestion : ce qu'en pensent les étudiants », Revue internationale de pédagogie de l'enseignement supérieur [En ligne], 30(1) | 2014, mis en ligne le 07 avril 2014, consulté le 08 septembre 2020. URL : http://journals.openedition.org/ripes/784 DOI : https://doi.org/10.4000/ripes.784

Ce document a été généré automatiquement le 8 septembre 2020

Article L.111-1 du Code de la propriété intellectuelle. 


\section{L'apprentissage hybride en sciences de gestion : ce qu'en pensent les étudiants}

Jean-Pierre Béchard, Catherine Carré, Gabriella Frankel, Zoffirath Dissou, Céline Bazinet, Gilles Cazabon et Yves Goyette

\section{Introduction}

1 Comme dans nombre de champs professionnels, la gestion est aux prises avec de multiples enjeux qui se reflètent indéniablement dans ses enseignements à tous les niveaux de formation. Dans le cadre de cet article, de type compte-rendu de pratique, nous présentons comment l'hybridation des enseignements par les technologies éducatives a eu un impact positif sur les perceptions des étudiants adultes inscrits dans des formations universitaires courtes (certificats de $1^{\mathrm{er}}$ cycle) d'une grande école de gestion nord-américaine. Nous introduisons tout d'abord le contexte de cette innovation pédagogique pour enchaîner avec le déploiement du projet-pilote. S'ensuit un travail d'évaluation dans une perspective holistique, avec un regard sur des variations possibles dans les résultats selon les sessions académiques et les modèles pédagogiques hybrides. Cet article débouche à la fois sur une réflexion institutionnelle et sur la présentation de quatre propositions reliées à un cadre théorique en émergence.

\section{La mise en contexte de l'innovation pédagogique}

Les technologies de l'information et de la communication en éducation font maintenant partie du paysage de l'enseignement supérieur, mais avec des variations importantes entre les enseignants-chercheurs, les établissements et même les pays (Depover, Karsenti \& Komis, 2008). Dans ce contexte de l'apprentissage distribué par la technologie, deux modèles marquent la littérature; le modèle de l'apprentissage 
complètement en ligne (online learning) et le modèle hybride (hybrid learning) qui mélange des activités en présentiel et des activités en ligne.

Plusieurs recherches tentent de cerner le phénomène de l'apprentissage en ligne où l'on se centre sur l'apprenant et les processus cognitifs tout en utilisant Internet comme plateforme de diffusion avec des applications technopédagogiques web 2.0. À titre d'exemple, certains travaux se penchent sur le travail des enseignants (Seok, 2008; Wilson, 2007), d'autres scrutent la qualité des dispositifs et des effets sur les étudiants et leurs apprentissages (Ebersole, 2005; Frydenberg, 2007; Khare \& Lam, 2008). Certaines recherches esquissent le portrait des étudiants dans ces dispositifs et des processus organisationnels nécessaires à une telle implantation (Leitner, 2008 ; Sandeen, 2008). Finalement, d'autres chercheurs se lancent dans une ébauche de théorisation de l'apprentissage en ligne (Anderson, 2008).

Parallèlement à ce courant de recherche majeur, plusieurs auteurs documentent le modèle de l'apprentissage hybride, initient quelques modèles de base ou proposent des solutions pour le futur des institutions d'enseignement supérieur et des entreprises (Aycock, Graham \& Kaleta, 2002; Dziuban, Hartman \& Moskal, 2004; Garrison \& Vaughan, 2008; Kelly, 2007; Masson, MacNeill, Murphy \& Ross, 2008; Morote, Wittmann \& Kelly, 2007; Stacey \& Gerbic, 2008). L'apprentissage hybride, dispositif pédagogique qui allie les avantages du présentiel à la flexibilité du web, amène à réduire les heures de classe en présence des étudiants et à leur proposer des activités individuelles et collaboratives avec des outils en ligne spécifiques, et ce, en mode synchrone ou asynchrone. En conséquence, ce scénario engage les enseignants à reconcevoir leurs cours de façon à permettre un apprentissage en profondeur en classe et hors classe, individuellement et en équipe.

5 D'autres travaux ont plutôt cherché à comprendre le phénomène de l'apprentissage hybride dans son ensemble, particulièrement dans l'enseignement supérieur américain (Allen, Seaman, \& Garrett, 2007 ; Diaz \& Brown, 2010), pendant que d'autres chercheurs ont scruté la qualité de l'apprentissage dans un tel dispositif pédagogique (Ginns \& Ellis, 2007), ou le niveau épistémologique et méthodologique des recherches dans ce domaine (Bliuc, Goodyear \& Ellis, 2007). Finalement, s'appuyant davantage sur des points de vue et des essais, certains auteurs se sont risqués à prévoir ce que pourrait devenir l'apprentissage hybride dans un futur rapproché, tant en éducation qu'en formation dans les organisations (Bonk, Kim \& Zeng, 2006 ; Garrison \& Kanuka, 2004 ; Kim, Bonk \& Oh, 2008 ; Vaughan, 2007).

Dans l'enseignement de la gestion, les recherches empruntent ce même genre de découpage, soit l'analyse d'expériences sur le terrain (Arbaugh, Godfrey, Johnson, Leisen Pollack, Niendorf \& Wresch, 2009 ; Noblet, 2011), soit la mesure des attitudes et des perceptions des étudiants en situation pédagogique hybride (Hwang \& Arbaugh, 2009 ; Lopez-Perez, Perez-Lopez, \& Rodriguez-Arisa, 2011), soit l'évaluation de la qualité de la recherche dans le domaine (Arbaugh et al., 2009 ; Arbaugh, Desai, Rau \& Sridhar 2011), pour terminer sur un effort de prospective de ce modèle hybride pour les écoles et facultés de gestion (Thomas, 2007). De cette littérature, il ressort que la définition de l'apprentissage hybride n'est pas toujours claire, que le niveau de sophistication méthodologique des recherches est peu élevé, qu'une approche holistique de ce type de recherche est nécessaire et qu'il y a peu de consensus sur les variables clés en jeu dans un tel phénomène. 
7 Dans le cadre de cet article, nous voulons dépasser la confusion entourant la définition de l'apprentissage hybride. Graham (2006) mentionne que ce concept réfère soit à une combinaison de modalités technopédagogiques ou de méthodes pédagogiques, ou soit à une combinaison de modalités d'enseignement en classe et en ligne. C'est à cette dernière signification que nous nous référons quand nous parlons d'apprentissage hybride. Ainsi, selon Laster, Otte, Picciano et Sorg (2005), un cours selon le modèle de l'apprentissage hybride est celui qui intègre des activités en ligne avec des activités en classe de façon planifiée et pédagogique et dont la proportion des activités en classe (définie par l'institution) est remplacée par des activités en ligne. D'après les travaux du Sloan Consortium (Picciano \& Dziuban, 2007), un cours hybride contient entre $30 \%$ et $79 \%$ d'activités en ligne, comparativement à un cours en ligne $(80 \%$ des activités sont en ligne) ou un cours enrichi par les technologies éducatives ( $1 \%$ à $29 \%$ d'activités en ligne).

Dans la foulée d'un tel contexte théorique et face à un environnement concurrentiel de plus en plus exigeant perçu comme tel par les écoles et facultés de gestion angloaméricaines et francophones, nous avons conçu et implanté, durant les années universitaires 2009-2010 et 2010-2011, un projet d'apprentissage hybride dans les programmes de certificat (éducation permanente au $1^{\mathrm{er}}$ cycle) d'une grande école de gestion nord-américaine. Après avoir mis en contexte cette expérimentation pédagogique, nous avons analysé les données recueillies par sondage auprès des 805 étudiants inscrits dans 27 classes (dix cours distincts au total sur les deux ans). L'évaluation de cette innovation pédagogique permettra de dégager les principales leçons pédagogiques et organisationnelles, de jeter les bases d'une diffusion institutionnelle à plus grande échelle et de démarrer une conceptualisation du phénomène à l'étude.

\section{Le déploiement du projet pilote}

9 En juin 2008, la direction générale de l'établissement donne le mandat au nouveau directeur des programmes de certificat de faire une proposition concernant l'apprentissage en ligne. Appuyée par les enseignants et un comité aviseur ${ }^{1}$, la direction élabore un projet qui se concentre principalement sur l'apprentissage hybride. Quatre principes sont à la base de cette innovation: la technologie existante peut servir à hybrider les cours ; la motivation des enseignants est le premier moteur de l'innovation pédagogique; les cours sont adaptés en fonction des besoins des étudiants, de la matière à apprendre et des enseignants; la formation techno-pédagogique des étudiants et des enseignants est incontournable.

10 Pour mettre en œuvre ce projet, un comité de pilotage (un groupe restreint de six personnes issues du comité aviseur) s'est assuré de la bonne marche du déploiement durant les deux années du projet. Tous les enseignants ont bénéficié d'un accompagnement individuel et collectif de la part des conseillères pédagogiques de la direction de l'apprentissage et de l'innovation pédagogique de l'établissement, et ce, durant toute la période de l'implantation. Cette même équipe a aussi contribué à la construction du questionnaire ainsi qu'à la cueillette et à l'analyse de données pour documenter ce projet pédagogique. Mentionnons que des séances d'informationdémonstration des outils ont été proposées aux étudiants en début de chaque session. Le tableau 1 présente les deux modèles hybrides que nous avons expérimentés. Les 
premiers résultats de cette expérimentation ont fait l'objet de deux présentations orales devant les diverses instances pédagogiques de l'institution (hiver 2010 et 2011) ainsi que devant la communauté scientifique (Béchard, Blanc, Frankel, Carré \& Bazinet, 2010 ; Béchard, Bazinet, Carré, Frankel, Souli \& Goyette, 2011).

Tableau 1. Deux modèles hybrides au banc d'essai

\begin{tabular}{|c|c|c|}
\hline & $\begin{array}{l}\text { Modèle hybride } \\
\text { par séance }\end{array}$ & $\begin{array}{l}\text { Modèle hybride } \\
\text { par bloc de séances }\end{array}$ \\
\hline Séance 1 & $\begin{array}{c}\text { Apprentissage en } \\
\text { présentiel }\end{array}$ & Apprentissage en présentiel \\
\hline Séance 2 & $\begin{array}{l}\text { Apprentissage en } \\
\text { présentiel }\end{array}$ & Apprentissage en présentiel \\
\hline Séance 3 & Apprentissage en ligne & Apprentissage en ligne \\
\hline Séance 4 & $\begin{array}{l}\text { Apprentissage en } \\
\text { présentiel }\end{array}$ & Apprentissage en ligne \\
\hline Séance 5 & Apprentissage en ligne & Apprentissage en ligne \\
\hline Séance 6 & $\begin{array}{l}\text { Apprentissage en } \\
\text { présentiel }\end{array}$ & Apprentissage en présentiel \\
\hline Séance 7 & Apprentissage en ligne & Apprentissage en présentiel \\
\hline Séance 8 & $\begin{array}{l}\text { Apprentissage en } \\
\text { présentiel }\end{array}$ & Apprentissage en ligne \\
\hline Séance 9 & Apprentissage en ligne & Apprentissage en ligne \\
\hline Séance 10 & $\begin{array}{l}\text { Apprentissage en } \\
\text { présentiel }\end{array}$ & Apprentissage en ligne \\
\hline Séance 11 & Apprentissage en ligne & Apprentissage en présentiel \\
\hline Séance 12 & $\begin{array}{c}\text { Apprentissage en } \\
\text { présentiel }\end{array}$ & Apprentissage en présentiel \\
\hline
\end{tabular}

11 Plusieurs outils ont été utilisés dans les modèles par séance et par bloc de séances. Citons, par exemple, l'environnement numérique d'apprentissage de l'institution, des outils de classes virtuelles synchrones, des forums de discussion, des questionnaires automatisés, des outils de collaboration, sans compter des capsules de contenus préenregistrées et des ressources électroniques de la bibliothèque et du service de l'audiovisuel. Les enseignants ont été non seulement formés à ces moyens, mais ont surtout bénéficié d'une aide techno-pédagogique pour repenser leur cours. Mentionnons qu'aucun enseignant n'avait préalablement vécu dans un contexte d'apprentissage hybride de même que pour les responsables de l'implantation qui ont été invités à déployer beaucoup de flexibilité et d'innovation organisationnelle.

\section{L'évaluation de l'innovation pédagogique}

Tout au long de cette section, nous abordons l'évaluation de l'innovation pédagogique de façon globale en agrégeant l'ensemble des données, puis de façon plus spécifique, en comparant les résultats selon les sessions académiques et le modèle pédagogique hybride. Dans les deux cas, notre préoccupation est triple. Nous questionnons tout d'abord les raisons qui ont poussé les étudiants à s'inscrire à un cours hybride, puis leur degré d'appréciation du dispositif d'enseignement et d'apprentissage et nous terminons avec leurs perceptions des impacts d'une telle expérience sur leurs apprentissages. Fait 
important à noter, toutes les données présentées sont de nature descriptive et aucun traitement statistique n'a été appliqué. Mentionnons de plus que tous les énoncés du questionnaire ont été développés, non pas à partir d'une revue de littérature, mais plutôt des préoccupations des praticiens membres du comité de pilotage. Nous avions des questions, des doutes et des hésitations et nous voulions avoir un début de réponse pour ainsi progresser avec un peu plus de certitude dans ce projet institutionnel.

\subsection{Une appréciation globale des résultats}

Les tableaux 2 et 3 détaillent les cours hybrides selon les sessions, les titres de cours, les professeurs désignés, le nombre de répondants aux sondages (format papier ou en ligne à la fin des cours), le nombre d'étudiants inscrits et, finalement, le taux de réponse par cours. En ce qui concerne le modèle hybride par séance, 419 étudiants sur un total de 568 étudiants ont répondu à notre appel, tandis que dans le modèle hybride par bloc de séances, 168 étudiants sur un total de 237 étudiants ont fait de même. Au global, 587 étudiants sur les 805 inscrits ont répondu à l'évaluation pédagogique, pour un taux de réponse de $72,9 \%$.

Tableau 2. Taux de réponse par session et par cours - modèle par séance

\begin{tabular}{|c|c|c|c|c|c|c|}
\hline Sessions & Cour & s hybrides par séance & Enseignants & $\begin{array}{c}\mathrm{Nb} . \\
\text { répondants }\end{array}$ & $\begin{array}{l}\text { Nb. étudiants } \\
\text { inscrits }\end{array}$ & $\begin{array}{l}\text { Taux de } \\
\text { réponse }\end{array}$ \\
\hline \multirow{3}{*}{ 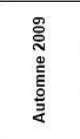 } & $\begin{array}{l}30- \\
403\end{array}$ & $\begin{array}{l}\text { Devenir une } \\
\text { organisation apprenante }\end{array}$ & Professeur $\mathrm{E}$ & 21 & 23 & $91,3 \%$ \\
\hline & $\begin{array}{l}30- \\
420\end{array}$ & $\begin{array}{l}\text { Habiletés de } \\
\text { supervision }\end{array}$ & Professeur $F$ & 32 & 39 & $82,1 \%$ \\
\hline & $\begin{array}{l}30- \\
410\end{array}$ & $\begin{array}{l}\text { Leadership et pratique } \\
\text { de direction }\end{array}$ & Professeur B & 26 & 38 & $68,4 \%$ \\
\hline \multirow{2}{*}{ 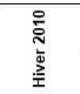 } & $\begin{array}{l}30- \\
403\end{array}$ & $\begin{array}{l}\text { Devenir une } \\
\text { organisation apprenante }\end{array}$ & Professeur $\mathrm{E}$ & 16 & 19 & $84,2 \%$ \\
\hline & $\begin{array}{l}30- \\
420\end{array}$ & $\begin{array}{l}\text { Habiletés de } \\
\text { supervision }\end{array}$ & Professeur 1 & 31 & 37 & $83,8 \%$ \\
\hline \multirow{7}{*}{ 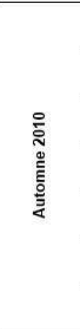 } & $\begin{array}{l}30- \\
503\end{array}$ & $\begin{array}{l}\text { Progiciels de gestion } \\
\text { intégrée }\end{array}$ & Professeur $\mathbf{G}$ & 26 & 31 & $83,9 \%$ \\
\hline & $\begin{array}{l}30- \\
100\end{array}$ & Introduction marketing & Professeur $\mathrm{H}$ & 29 & 40 & $72,5 \%$ \\
\hline & $\begin{array}{l}30- \\
460\end{array}$ & Habiletés politiques & Professeur I & 26 & 35 & $74,3 \%$ \\
\hline & $\begin{array}{l}30- \\
403\end{array}$ & $\begin{array}{l}\text { Devenir une } \\
\text { organisation apprenante }\end{array}$ & Professeur $\mathrm{E}$ & 9 & 13 & $69,2 \%$ \\
\hline & $\begin{array}{l}30- \\
411\end{array}$ & Gestion du changement & Professeur $\mathrm{J}$ & 14 & 21 & $66,7 \%$ \\
\hline & $\begin{array}{l}30- \\
411\end{array}$ & Gestion du changement & Professeur $\mathrm{J}$ & 9 & 17 & $52,9 \%$ \\
\hline & $\begin{array}{l}30- \\
420\end{array}$ & $\begin{array}{l}\text { Habiletés de } \\
\text { supervision }\end{array}$ & Professeur $F$ & 11 & 17 & $64,7 \%$ \\
\hline \multirow{7}{*}{ 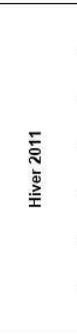 } & $\begin{array}{l}30- \\
503\end{array}$ & $\begin{array}{l}\text { Progiciels de gestion } \\
\text { intégree }\end{array}$ & Professeur G & 28 & 34 & $82,4 \%$ \\
\hline & $\begin{array}{l}30- \\
100\end{array}$ & Introduction marketing & Professeur $\mathrm{H}$ & 29 & 46 & $63,0 \%$ \\
\hline & $\begin{array}{l}30- \\
460\end{array}$ & Habiletés politiques & Professeur 1 & 27 & 40 & $67,5 \%$ \\
\hline & $\begin{array}{l}30- \\
403\end{array}$ & $\begin{array}{l}\text { Devenir une } \\
\text { organisation apprenante }\end{array}$ & Professeur $E$ & 13 & 15 & $86,7 \%$ \\
\hline & $\begin{array}{l}30- \\
411\end{array}$ & Gestion du changement & Professeur $\mathrm{J}$ & 37 & 50 & $74,0 \%$ \\
\hline & $\begin{array}{l}30- \\
410\end{array}$ & $\begin{array}{l}\text { Leadership et pratique } \\
\text { de direction }\end{array}$ & Professeur B & 10 & 19 & $52,6 \%$ \\
\hline & $\begin{array}{l}30- \\
420\end{array}$ & $\begin{array}{l}\text { Habiletés de } \\
\text { supervision }\end{array}$ & Professeur $F$ & 25 & 34 & $73,5 \%$ \\
\hline \multicolumn{4}{|c|}{ Nombre total de répondants } & 419 & 568 & $73,8 \%$ \\
\hline
\end{tabular}


Tableau 3. Taux de réponse par session et par cours - modèle par bloc de séances

\begin{tabular}{|c|c|c|c|c|c|c|}
\hline Sessions & Cours & $\begin{array}{l}\text { hybrides par bloc de } \\
\text { séances }\end{array}$ & Enseignants & $\begin{array}{l}\mathrm{Nb} . \\
\text { répondants }\end{array}$ & $\begin{array}{l}\text { Nb. } \\
\text { étudiants } \\
\text { inscrits }\end{array}$ & $\begin{array}{l}\text { Taux de } \\
\text { réponse }\end{array}$ \\
\hline 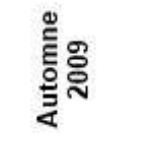 & $30-530$ & $\begin{array}{l}\text { Fondements de la } \\
\text { qualité totale }\end{array}$ & Professeur A & 27 & 34 & $79,4 \%$ \\
\hline \multirow{2}{*}{ 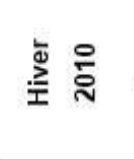 } & $30-410$ & $\begin{array}{l}\text { Leadership et } \\
\text { pratique de direction }\end{array}$ & Professeur B & 23 & 25 & $92,0 \%$ \\
\hline & $30-530$ & $\begin{array}{l}\text { Fondements de la } \\
\text { qualité totale }\end{array}$ & Professeur A & 33 & 47 & $70,2 \%$ \\
\hline \multirow{3}{*}{ 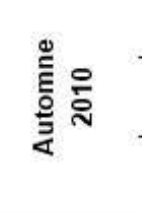 } & $30-433$ & Sociologie du travail & Professeur C & 14 & 23 & $60,9 \%$ \\
\hline & $30-410$ & $\begin{array}{l}\text { Leadership et } \\
\text { pratique de direction }\end{array}$ & Professeur B & 15 & 25 & $60,0 \%$ \\
\hline & $30-530$ & $\begin{array}{l}\text { Fondements de la } \\
\text { qualité totale }\end{array}$ & Professeur A & 13 & 19 & $68,4 \%$ \\
\hline \multirow{2}{*}{ 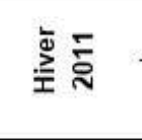 } & $30-433$ & Sociologie du travail & Professeur C & 14 & 16 & $87,5 \%$ \\
\hline & $30-530$ & $\begin{array}{l}\text { Fondements de la } \\
\text { qualité totale }\end{array}$ & Professeur D & 29 & 48 & $60,4 \%$ \\
\hline \multicolumn{4}{|c|}{ Nombre total de répondants } & 168 & 237 & $70,89 \%$ \\
\hline
\end{tabular}

Le tableau 4 fait état du profil sociodémographique des 587 répondants de l'étude. Le portrait type se lit comme suit: l'étudiant est un homme $(52,4 \%)$, est âgé majoritairement entre 26 et 35 ans, est un travailleur à temps plein ( $71,3 \%)$, est inscrit à un seul cours $(36,6 \%)$ et réside à 20 kilomètres et plus du campus $(44,3 \%)$. On peut avancer, sans crainte de se tromper que ce profil est très similaire au profil des étudiants adultes qui fréquentent habituellement nos programmes courts de $1^{\mathrm{er}}$ cycle en gestion. Nous ne sommes donc pas devant un segment non représentatif de la population générale. 
Tableau 4. Profil de tous les répondants de l'enquête

\begin{tabular}{|c|c|c|}
\hline \multicolumn{3}{|l|}{ Agrégation globale } \\
\hline \multicolumn{3}{|l|}{ Profil } \\
\hline \multirow{2}{*}{ Sexe } & Hommes & $52,4 \%$ \\
\hline & Femmes & $47,6 \%$ \\
\hline \multirow{5}{*}{ Äge } & $18-25$ ans & $16,7 \%$ \\
\hline & $26-35$ ans & $49,5 \%$ \\
\hline & $36-45$ ans & $25,8 \%$ \\
\hline & $46-55$ ans & $6,9 \%$ \\
\hline & 56 ans et plus & $1,2 \%$ \\
\hline \multirow{3}{*}{ Situation professionnelle } & Travailleur à temps plein & $71,3 \%$ \\
\hline & Travailleur à temps partiel & $12,6 \%$ \\
\hline & Sans emploi & $16,1 \%$ \\
\hline \multirow{4}{*}{$\begin{array}{l}\text { Nombre de cours suivis } \\
\text { durant la session }\end{array}$} & Un & $36,6 \%$ \\
\hline & Deux & $20,9 \%$ \\
\hline & Trois & $13,0 \%$ \\
\hline & Quatre et plus & $29,6 \%$ \\
\hline \multirow{4}{*}{$\begin{array}{l}\text { Distance par rapport au } \\
\text { campus }\end{array}$} & $0-5 \mathrm{~km}$ & $8,9 \%$ \\
\hline & $6-10 \mathrm{~km}$ & $18,7 \%$ \\
\hline & $11-20 \mathrm{~km}$ & $28,1 \%$ \\
\hline & $20 \mathrm{~km}$ et plus & $44,3 \%$ \\
\hline
\end{tabular}

15 La première question posée aux étudiants concernait leurs raisons de s'inscrire à un cours hybride. La figure 1 fait état de leurs réponses. La flexibilité dans les déplacements est de loin la plus importante raison $(30,5 \%)$, suivie de la flexibilité des horaires $(19,1 \%)$ et de la curiosité pour cette innovation dans notre établissement $(16,1 \%)$. La quatrième raison a trait à la conciliation travail-famille-études $(7,4 \%)$ et le contenu du cours $(7,3 \%)$. La technologie vient loin derrière $(2,8 \%)$. 
Figure 1. Raisons des étudiants de s'inscrire à un cours hybride

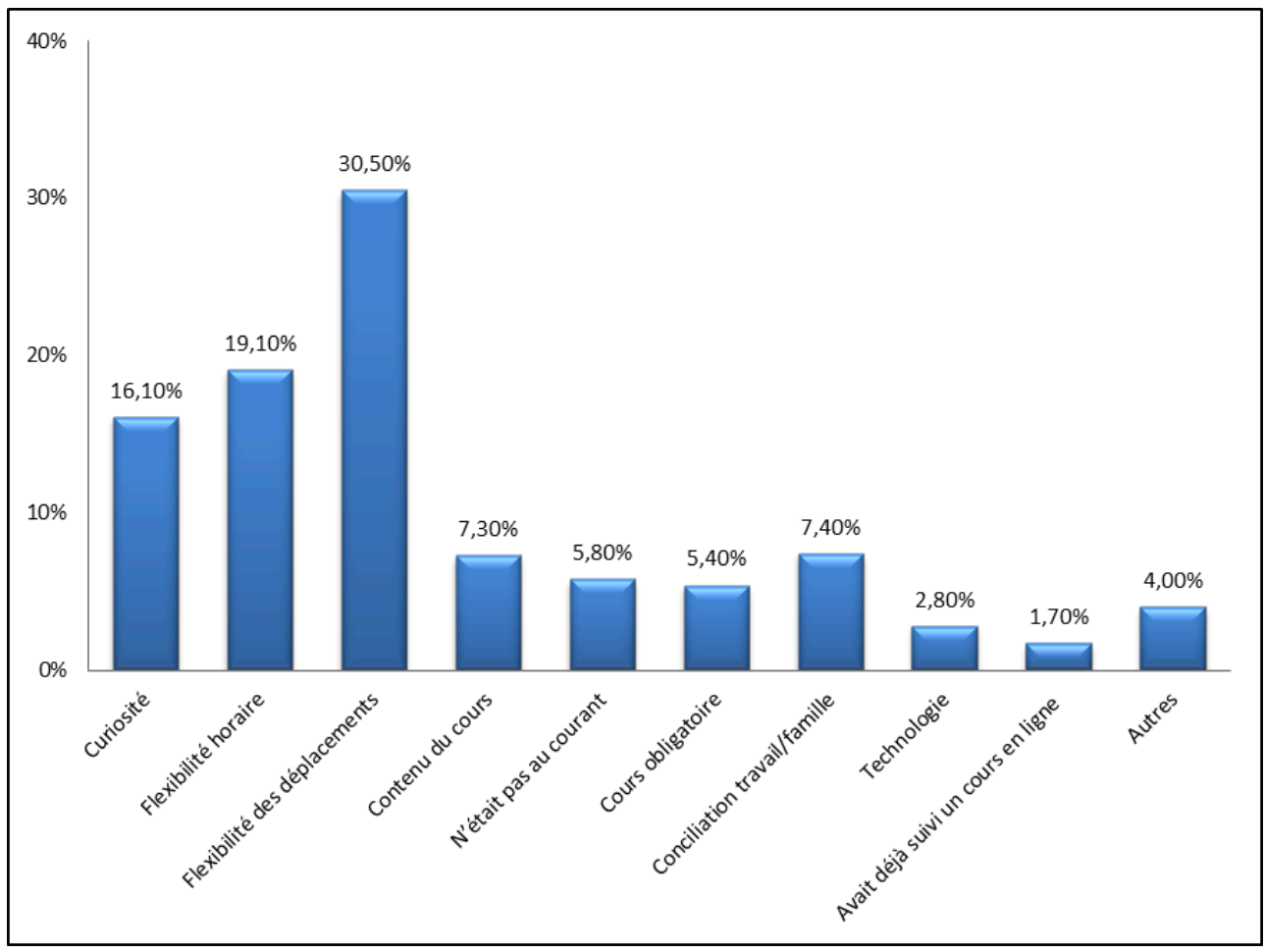

Sur le plan pédagogique, les réponses du tableau 5 sont intéressantes. L'appréciation générale est très forte (plus de $90 \%$ ) eu égard au dispositif et à la possibilité de planifier les apprentissages selon leur propre rythme. Dans près de $80 \%$ des cas, les étudiants n'ont pas eu de difficulté à gérer leur temps de travail et ne se sont pas sentis isolés pendant les séances de classe virtuelle.

Tableau 5. Appréciation générale des cours hybrides

\begin{tabular}{|c|c|c|c|c|}
\hline \multicolumn{5}{|l|}{ Agrégation globale } \\
\hline \multicolumn{2}{|c|}{ Appréciation générale du cours } & \multirow{2}{*}{$\begin{array}{c}\begin{array}{c}\text { Ne sait } \\
\text { pas }\end{array} \\
0,5 \%\end{array}$} & \multirow{2}{*}{$\begin{array}{c}\begin{array}{c}\text { En } \\
\text { accord }\end{array} \\
90,1 \%\end{array}$} & \multirow{2}{*}{$\begin{array}{c}\text { En } \\
\text { désaccord } \\
9,4 \%\end{array}$} \\
\hline $\begin{array}{l}\text { Satisfaction par } \\
\text { rapport au } \\
\text { dispositif }\end{array}$ & $\begin{array}{l}\text { J'ai trouvé satisfaisant d'alterner une } \\
\text { séance en classe et une séance (ou } \\
\text { bloc de séances) d'apprentissage à } \\
\text { distance }\end{array}$ & & & \\
\hline $\begin{array}{l}\text { Planification de } \\
\text { l'apprentissage }\end{array}$ & $\begin{array}{l}\text { J'ai été en mesure de planifier mon } \\
\text { apprentissage au rythme que je } \\
\text { souhaitais durant ce cours avec } \\
\text { apprentissage en ligne }\end{array}$ & $1,2 \%$ & $90,1 \%$ & $8,7 \%$ \\
\hline $\begin{array}{l}\text { Gestion du temps } \\
\text { de travail }\end{array}$ & $\begin{array}{l}\text { J'ai eu de la difficulté à gérer mon } \\
\text { temps de travail durant ce cours avec } \\
\text { apprentissage en ligne }\end{array}$ & $0,7 \%$ & $20,5 \%$ & $78,8 \%$ \\
\hline $\begin{array}{l}\text { Sentiment } \\
\text { d'isolement }\end{array}$ & $\begin{array}{l}\text { Je me suis senti isolé pendant les } \\
\text { séances (ou les blocs) d'apprentissage } \\
\text { à distance }\end{array}$ & $0,8 \%$ & $20,3 \%$ & $78,9 \%$ \\
\hline
\end{tabular}


Quand on creuse la question des impacts perçus de cette innovation pédagogique sur les apprentissages des étudiants, leurs réponses portent à réflexion comme en témoigne le tableau 6. Tout d'abord, les résultats font état de perceptions majoritairement similaires à celles que les étudiants vivent dans les classes en présentiel (dominance du score "également»). Par ailleurs, dans la colonne des réponses "plus", les chiffres expriment une plus grande motivation $(34,8 \%)$, un meilleur niveau d'activité $(26,9 \%)$, une plus grande charge de travail $(17,8 \%)$ et de meilleurs résultats d'apprentissage $(18,7 \%)$ que s'ils avaient suivi le cours entièrement en classe. Fait important à noter, le niveau d'interactions perçu est aussi celui qui a le score «moins » le plus élevé, faisant état d'une polarisation des réponses face à cette question. On peut se questionner sur la nature du dispositif hybride. Le fait que l'enseignant est physiquement moins présent et qu'une partie de la matière doit être apprise de façon autonome (lors des séances en ligne en mode asynchrone) nous porte à croire que l'étudiant s'engage davantage dans le cours hybride que dans un cours complètement en présentiel.

Tableau 6. Perceptions des impacts des cours hybrides

\begin{tabular}{|c|c|c|c|c|}
\hline \multicolumn{5}{|l|}{ Agrégation globale } \\
\hline \multicolumn{2}{|c|}{ Appréciation générale du cours } & \multirow{2}{*}{$\begin{array}{l}\text { Plus } \\
34,8 \%\end{array}$} & \multirow{2}{*}{$\begin{array}{c}\text { Également } \\
46,7 \%\end{array}$} & \multirow{2}{*}{$\begin{array}{l}\text { Moins } \\
18,5 \%\end{array}$} \\
\hline Motivation & $\begin{array}{l}\text { Je pense que j'ai été } \\
\text { dans ce cours avec apprentissage en } \\
\text { ligne que si j'avais pu le suivre } \\
\text { entièrement en classe }\end{array}$ & & & \\
\hline $\begin{array}{l}\text { Niveau } \\
\text { d'interactions }\end{array}$ & $\begin{array}{l}\text { Je pense que j'ai été } \text { actif dans }_{\text {ce cours avec apprentissage en ligne }} \\
\text { que si javais pu le suivre entièrement } \\
\text { en classe }\end{array}$ & $26,9 \%$ & $47,3 \%$ & $25,9 \%$ \\
\hline Charge de travail & $\begin{array}{l}\text { Je pense que j'ai eu une charge de } \\
\text { travail importante dans ce } \\
\text { cours avec apprentissage en ligne que } \\
\text { si j'avais pu le suivre entièrement en } \\
\text { classe }\end{array}$ & $17,8 \%$ & $63,1 \%$ & $19,0 \%$ \\
\hline Apprentissages & $\begin{array}{l}\text { Je pense que j'ai } \\
\text { ce cours avec apprentissage en ligne } \\
\text { que si j'avais pu le suivre entièrement } \\
\text { en classe }\end{array}$ & $18,7 \%$ & $63,8 \%$ & $17,5 \%$ \\
\hline
\end{tabular}

18 Peut-on déceler des variations dans les réponses tout au long des quatre sessions de notre expérimentation pédagogique et aussi selon les deux modèles pédagogiques? La prochaine section fait le point sur ces dimensions.

\subsection{La comparaison des résultats dans le temps et selon le modèle pédagogique}

L'analyse des résultats en fonction des sessions de l'expérimentation propose des nuances intéressantes. Sur le plan des raisons de s'inscrire à un cours hybride, c'est la flexibilité dans les déplacements qui prend de plus en plus d'importance (de $24,7 \%$ à l'automne 2009 à 32,9\% à l'hiver 2011); vient ensuite la flexibilité des horaires (de $16,0 \%$ à 19,6\%), reléguant au troisième rang la curiosité (de 18,0 \% à 16,7\%). Par 
ailleurs, la conciliation travail-famille-études est une raison qui semble perdre de l'importance au fil des sessions (de $8,7 \%$ à 5,4 \%). Quand on compare les réponses en fonction des modèles pédagogiques en alternance et thématique, aucune différence ne ressort, les raisons et leur importance relative étant identiques.

20 Au chapitre de l'appréciation générale des cours hybrides selon les sessions, la figure 2 présente les réponses des étudiants. Il semble que les pourcentages de réponses sous la rubrique « en accord " avec la satisfaction par rapport au dispositif et la planification de l'apprentissage soient très élevés d'une part (plus de $90 \%$ en général) et comparables au fil des quatre sessions. Par contre, la gestion du temps de travail est passée de $26,4 \%$ à 17,0\%, signifiant une moins grande difficulté chez les étudiants à s'organiser et un plus grand sentiment d'isolement à partir de l'hiver 2010 jusqu'à l'hiver 2011 (de 12,6\% à 24,9\%, mais sans jamais atteindre les sommets de l'automne de 2009 (29,2 \%).

Figure 2. Évolution de l'appréciation générale du cours en pourcentage des étudiants ayant répondu "En accord"

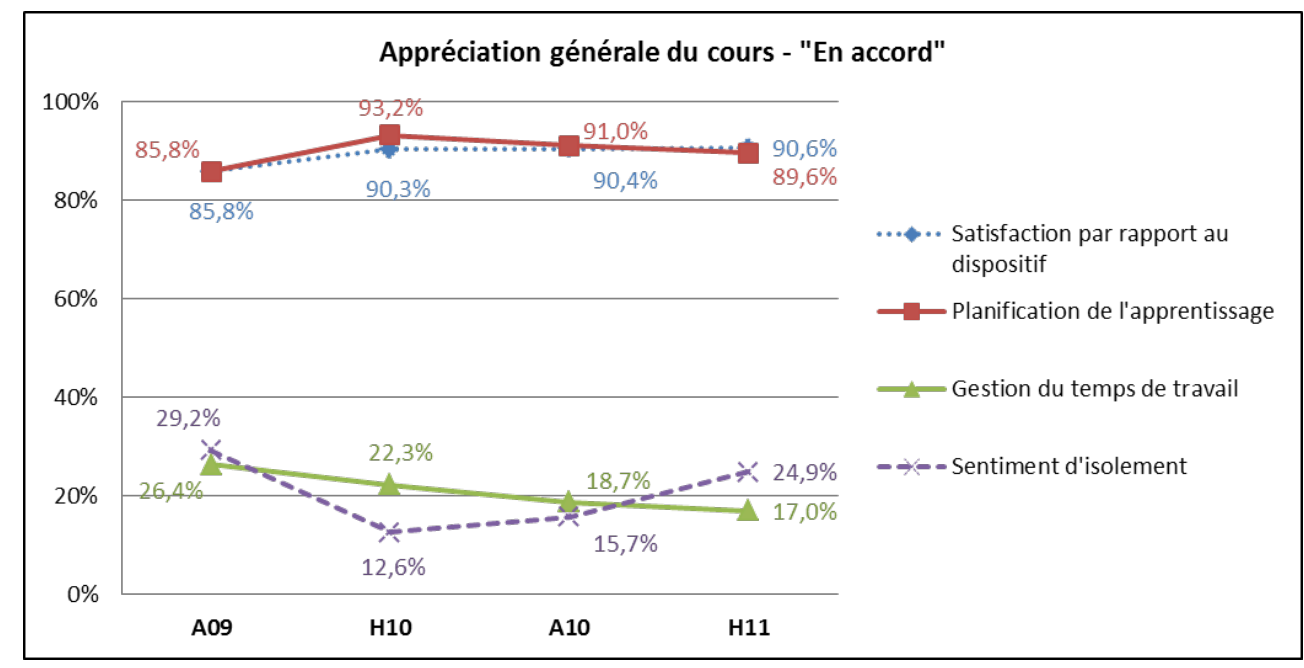

21 Quand on examine les résultats de l'appréciation générale selon les modèles pédagogiques à l'étude, nous observons trois différences. 
Figure 3. Appréciation générale du cours selon les modèles hybrides

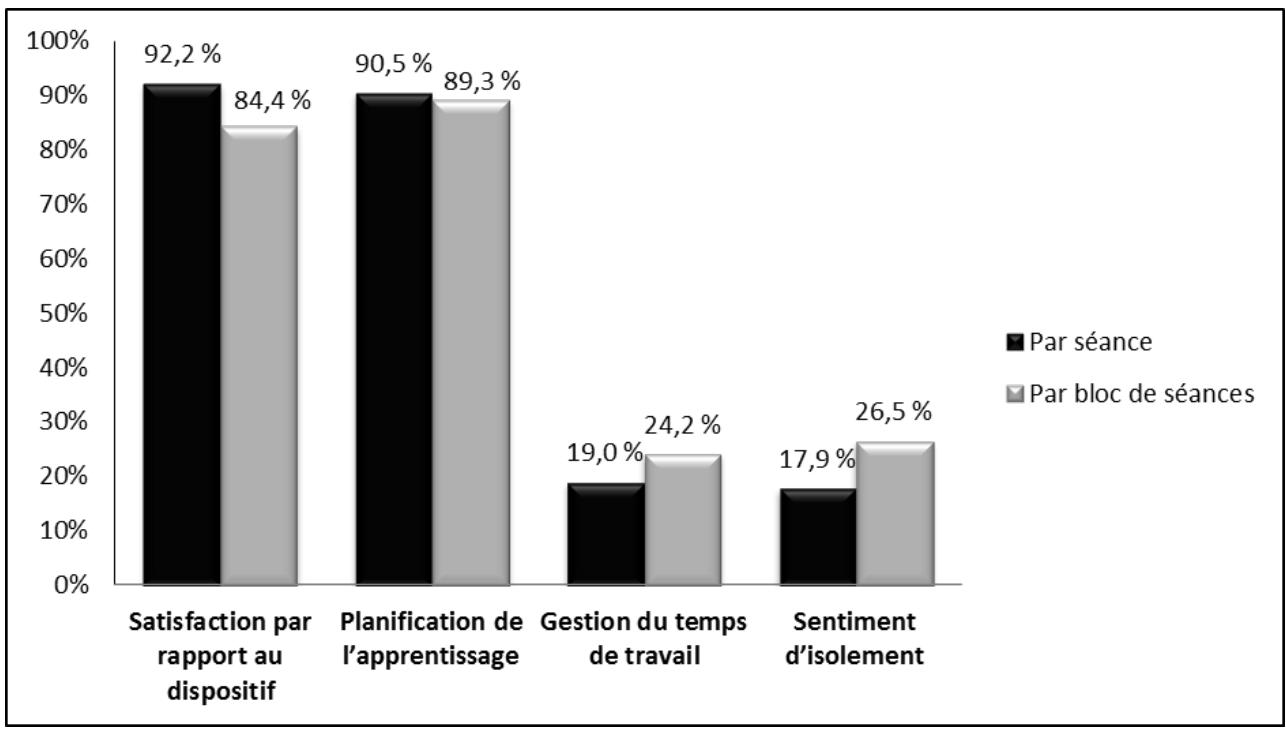

Tout d'abord, le modèle par séance semble susciter plus d'accords sur le plan de la satisfaction du dispositif que le modèle par bloc de séances (respectivement 92,2\% et $84,4 \%$ ), même si les deux taux de réponse sont très élevés. Du côté de la gestion du temps de travail, le modèle par séance dépasse le modèle par bloc de séances $(24,2 \%$ et $19,0 \%$ ). Même chose du côté du sentiment d'isolement avec des scores respectifs de $26,5 \%$ et $17,9 \%$. D'après les réponses des étudiants, seule celle qui a trait à la planification de l'apprentissage selon son propre rythme semble être identique. Poursuivons notre investigation sur les perceptions des étudiants par rapport aux impacts sur les apprentissages, et ce, en fonction des sessions et des modèles hybrides choisis. Le tableau 7 présente les réponses des étudiants selon les quatre énoncés à compléter.

Tableau 7. Perceptions des impacts selon les sessions et selon les réponses « également » et " plus » $(27$ cours $)$

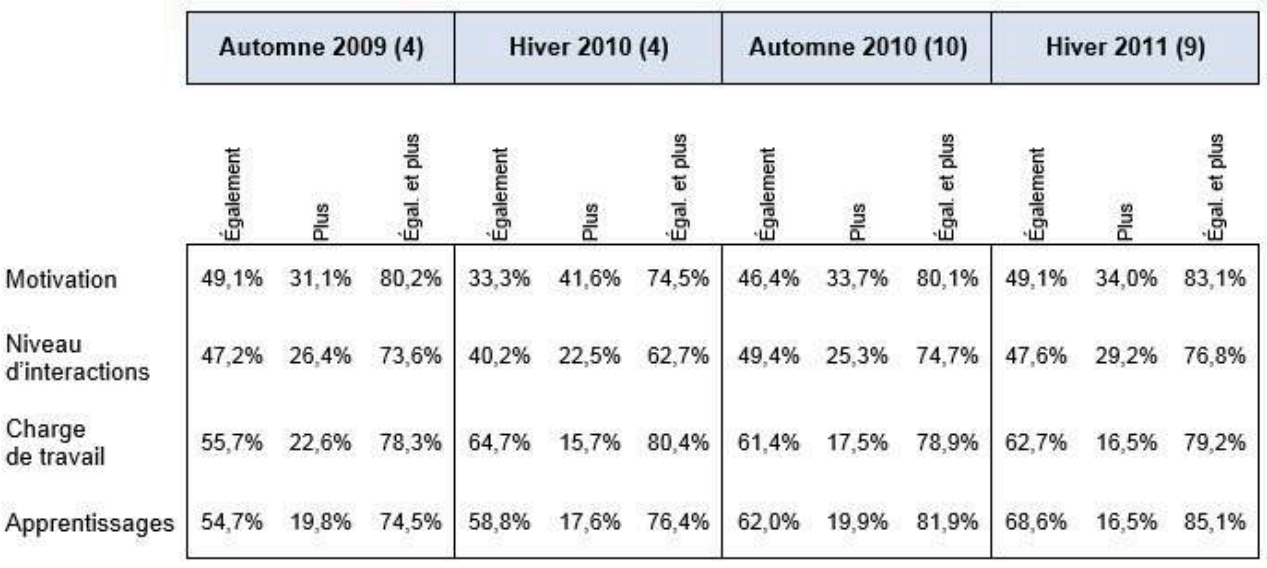

$23 \mathrm{Au}$ fil du temps sous observation, les étudiants ont eu cette perception d'être «également et plus» motivés avec un sommet de 83,1\% à l'hiver 2011. Même observation pour le niveau d'activité perçu qui semble être en croissance (à l'exception des réponses de l'hiver 2010). La charge de travail semble relativement stable selon les 
périodes. Mais, les perceptions des apprentissages ont fait un bond, passant de 74,5 à $85,1 \%$.

Finalement, le tableau 8 étale les réponses des étudiants au chapitre des impacts perçus selon les modèles pédagogiques. Quand on agrège les réponses « également » et " plus » associés aux impacts des dispositifs hybrides sur les apprentissages, les résultats sont marquants. Sur toutes les questions (motivation, niveau d'activité, charge de travail et résultats d'apprentissage), le modèle pédagogique par séance semble supérieur au modèle pédagogique par bloc de séances. De plus, nos rencontres informelles avec les enseignants ont renforcé cette idée que le modèle par bloc de séances semble plus difficile pour les étudiants adultes. Ces derniers retournent aux études après une période de travail plus ou moins longue et n'ont pas toujours retrouvé ou acquis les bonnes habitudes associées au métier d'étudiant. Rappelons toutefois qu'aucun traitement statistique n'a été appliqué, ce qui nous invite à la prudence dans l'analyse des résultats.

Tableau 8. Perceptions des impacts selon les modèles pédagogiques

\begin{tabular}{l|ccc|ccc|}
\hline \multicolumn{1}{c|}{} & \multicolumn{4}{|c|}{ Modèle hybride par séance } & \multicolumn{2}{c|}{ Modèle hybride par bloc de séances } \\
\hline & Également & Plus & $\begin{array}{c}\text { Également } \\
\text { et plus }\end{array}$ & Également & Plus & $\begin{array}{c}\text { Également } \\
\text { et plus }\end{array}$ \\
\cline { 2 - 7 } & $\begin{array}{l}\text { Motivation } \\
47,4 \%\end{array}$ & $36,7 \%$ & $84,1 \%$ & $42,8 \%$ & $28,9 \%$ & $71,7 \%$ \\
$\begin{array}{l}\text { Niveau } \\
\text { d'interactions }\end{array}$ & $48,1 \%$ & $28,0 \%$ & $76,1 \%$ & $43,5 \%$ & $22,6 \%$ & $66,1 \%$ \\
$\begin{array}{l}\text { Charge } \\
\text { de travail }\end{array}$ & $64,0 \%$ & $19,0 \%$ & $83,0 \%$ & $54,8 \%$ & $17,1 \%$ & $71,9 \%$ \\
Apprentissages & $65,2 \%$ & $18,0 \%$ & $83,2 \%$ & $56,3 \%$ & $18,4 \%$ & $74,7 \%$ \\
\hline
\end{tabular}

La section suivante se penche sur la suite de nos réflexions pédagogiques et organisationnelles et jette les bases d'une première conceptualisation.

\section{Quelques leçons empiriques et conceptuelles à retenir}

L'évaluation de cette innovation pédagogique, à partir des perceptions des étudiants qui ont vécu le dispositif hybride, a permis de déceler quelques éléments clés :

- Les raisons de s'inscrire à un cours hybride réfèrent principalement à la flexibilité dans les déplacements et les horaires, et ce, peu importe la session et le modèle ; ceci semble aller de pair avec les besoins des étudiants adultes de nos programmes ;

- L'appréciation générale des cours hybrides est très élevée, malgré une certaine difficulté à gérer le temps de travail et le fait de vivre un sentiment d'isolement qui varie quelque peu dans le temps et selon les modèles pédagogiques ;

- À mesure que les sessions avancent, les impacts positifs de cette expérimentation ressortent de plus en plus et sous toutes les dimensions sondées ;

- Le modèle pédagogique par séance semble, à nos yeux un dispositif plus adapté à notre clientèle d'adultes en sciences de gestion. 

conforte dans la démarche que nous avons entreprise. Par exemple, à l'Université du Wisconsin Milwaukee, Aycock, Graham, et Kaleta (2002) ont identifié dix leçons qu'ils ont dégagées de l'hybridation de certains de leurs cours.

1. Il n'y a pas d'approche standard de l'apprentissage hybride ;

2. Le redesign d'un cours traditionnel prend du temps;

3. Commencer petit et garder cela simple ;

4. Le redesign est la clé la plus efficace pour intégrer les séances en face à face avec les séances en ligne ;

5. Les cours hybrides facilitent l'interaction entre les étudiants et entre l'enseignant et les étudiants ;

6. Les étudiants ne saisissent pas rapidement le concept d'hybridation des cours ;

7. La flexibilité du temps apporté par l'hybridation est universellement populaire ;

8. La technologie n'est pas un obstacle significatif ;

9. Développer un cours hybride fait appel à un processus collégial ;

10. Les enseignants et les étudiants aiment le modèle hybride.

Dans une autre expérience à l'Université de Calgary au Canada, Garrison et Vaughan (2008) ont sondé les perceptions des étudiants ayant participé à l'un des huit cours hybrides mis sur pied. En résumé, ce que les étudiants ont le plus apprécié est le travail en équipe, les discussions en face à face et en ligne, l'augmentation des interactions entre les étudiants et les enseignants et les ressources disponibles en ligne. Ces mêmes étudiants ont, par contre, moins apprécié le manque d'attentes claires et de lignes directrices, les composantes en ligne, les discussions en ligne, l'approche en autoformation et l'augmentation de la charge de travail.

En fait, l'évaluation de notre expérimentation pédagogique (qui a aussi été menée auprès des enseignants, mais dont les résultats ne font pas partie de cet article) a créé la légitimité pour accroître la diffusion de cette approche, non seulement dans les programmes de certificat (éducation permanente au $1^{\text {er }}$ cycle universitaire en gestion), mais aussi dans les cycles professionnels supérieurs de notre institution. Face à une demande de plus en plus importante de flexibilité de la part des apprenants adultes, cette approche semble une tendance lourde dans les prochaines années. De plus, l'apprentissage hybride n'est plus perçu comme une étape intermédiaire entre le présentiel et le $100 \%$ en ligne, mais plutôt comme une stratégie institutionnelle autonome tant pour les clientèles actuelles que pour les clientèles futures adultes. Ceci aura comme conséquence de mieux réfléchir à la qualité des apprentissages en contexte d'hybridité, tel que le suggèrent les travaux consacrés aux classes virtuelles synchrones (Clark \& Kwinn, 2007 ; MacDonald, 2008).

Sur le plan conceptuel, notre travail de terrain, couplé à une lecture attentive des recherches du domaine, amène à suggérer un cadre intégrateur en trois pôles (étudiants, enseignants, organisations) et à avancer quatre pistes de réflexion pour le futur. 


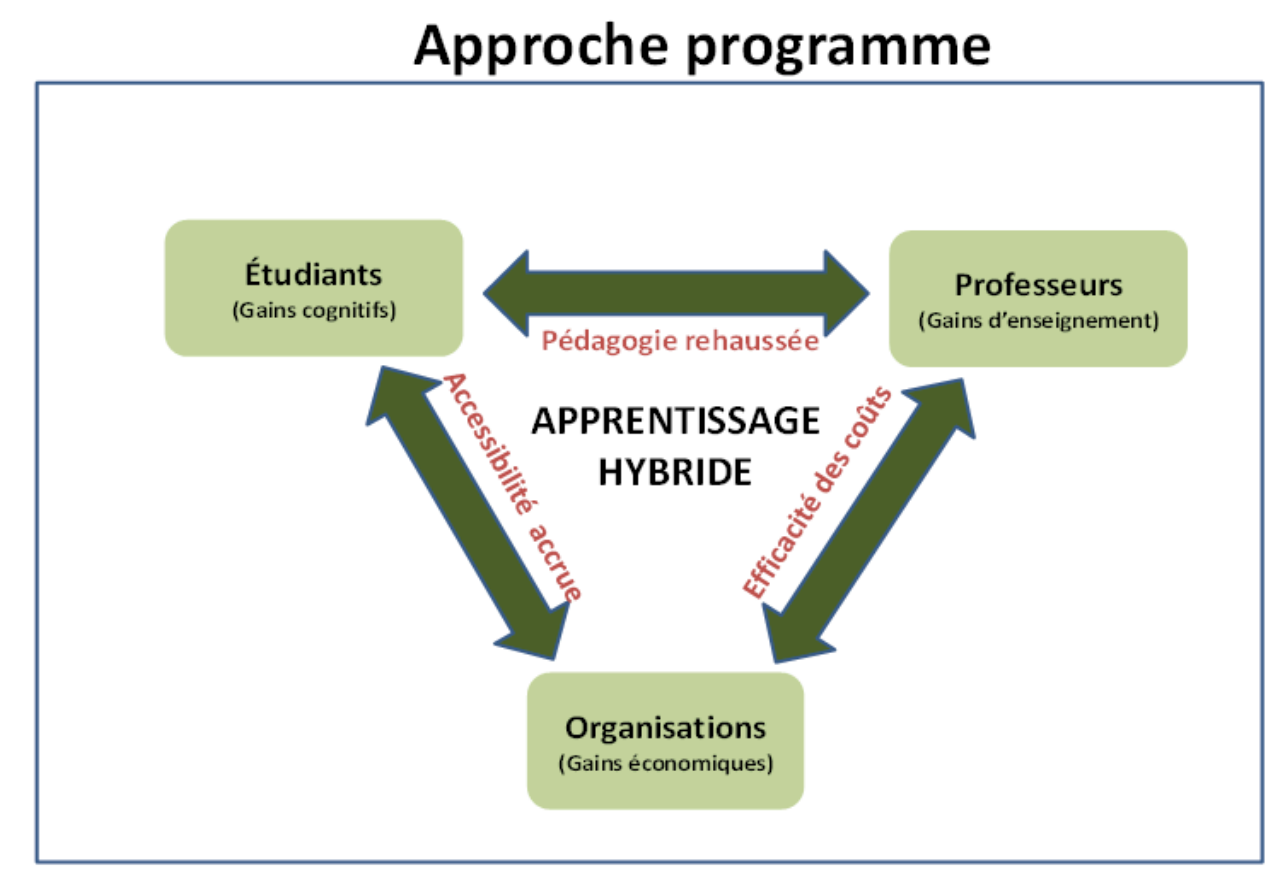

- Piste de réflexion 1 : Dans un contexte d'apprentissage hybride, il y aurait rehaussement de la pédagogie. Ce que nous avançons est que cette plus-value à la pédagogie serait influencée entre autres par le type d'hybridation, la perception positive de l'apprentissage hybride, la formation des enseignants et des étudiants aux technologies de l'information et de la communication en éducation, le redesign des cours, l'ajout de ressources humaines, matérielles et financières, sans oublier la capacité des étudiants et des enseignants à s'entraider ;

- Piste de réflexion 2 : Dans un contexte d'apprentissage hybride, il y aurait une accessibilité accrue aux études universitaires par la réponse aux besoins de flexibilité (temps et espace), des adultes dans notre cas, à une meilleure conciliation travail-famille-études et qui se traduirait pour l'organisation en gains économiques comme l'augmentation des inscriptions ;

- Piste de réflexion 3 : Dans un contexte d'apprentissage hybride, il y aurait une meilleure efficacité des coûts en améliorant la coordination des différents services de l'institution, en optimisant les environnements numériques d'apprentissage et en ayant la possibilité de réduire le nombre de sièges de classe physique ;

- Piste de réflexion 4 : Dans un contexte d'apprentissage hybride, le rehaussement de la pédagogie, l'accessibilité accrue aux études universitaires et une meilleure efficacité des coûts varieraient selon les contextes de programme, obligeant toute conceptualisation et implantation à tenir compte de la réalité de diffusion.

Bien évidemment, ces pistes de réflexion dépassent largement les résultats de la présente étude. Toutefois, nous pensons qu'elles sont porteuses d'idées dignes d'une pratique réflexive collective.

En conclusion, ce compte-rendu de pratique a tenté de pousser un peu plus loin la réflexion sur l'apprentissage hybride. Par une définition plus claire de ce qu'est l'apprentissage hybride, par une approche plus holistique de ce type de recherche et 
par une esquisse de conceptualisation de l'apprentissage hybride, nous avons voulu dépasser les limites de la littérature actuelle. Tout un travail reste à faire pour tester les pistes de réflexion dans différents programmes, scientifiques ou professionnels, de plusieurs institutions et auprès de l'ensemble des parties prenantes de l'innovation, comme le suggèrent les travaux de Noblet (2011).

Pour la suite des recherches sur l'apprentissage hybride, nous suggérons de construire un questionnaire à partir de la littérature existante sur la pédagogie en enseignement supérieur, de traiter les données statistiquement et de comparer les résultats des classes hybrides avec ceux des classes complètement en présentiel, tout en contrôlant idéalement les variables associées aux étudiants, aux enseignants et à la matière étudiée.

\section{BIBLIOGRAPHIE}

Allen, I.E., Seaman J. \& Garrett, R. (2007). Blending in. The extent and promise of blended education in United States. Repéré à http://sloanconsortium.org/publications/survey/blended06

Anderson, T. (2008). Theory and practice of online learning (2e éd.). Athabasca, AL: Athabasca University.

Arbaugh, J.B., Godfrey, M.R., Johnson, M., Leisen Pollack, B., Niendorf, B. \& Wresch, W. (2009). Research in online and blended learning in the business disciplines: Key findings and possible future directions. The Internet and Higher Education, 12, 71-87.

Arbaugh, J.B., Desai, A., Rau, B. \& Sridhar, B.S. (2010). A review of research on online and blended learning in the management disciplines-1994-2009. Organization Management Journal,7, 39-55.

Aycock, A., Graham, C. \& Kaleta, R. (2002). Lessons learned from the hybrid course project. Teaching with Technology Today, 8(6), 1-5.

Béchard, J-P., Blanc, P., Frankel, G., Carré, C. \& Bazinet, C. (2010, mai). Déploiement de l'apprentissage hybride en sciences de gestion : une première évaluation. Communication présentée au $26^{\mathrm{e}}$ congrès de l'AIPU, Rabat, Maroc.

Béchard, J-P., Bazinet, C., Carré, C., Frankel, G., Souli, F. \& Goyette, Y. (2011, mai). L'apprentissage hybride en sciences de gestion: ce qu'en pensent les étudiants. Communication présentée au $79^{\mathrm{e}}$ congrès de l'ACFAS, Sherbrooke, Québec.

Bliuc, A-M., Goodyear, P. \& Ellis, R.A. (2007). Research focus and methodological choices in studies into students' experiences of blended learning in higher education. The Internet and Higher Education, 10, 231-244.

Bonk, C.J., Kim, K-J. \& Zeng, T. (2006). Future directions of blended learning in higher education and workplace learning activities. Dans C.J. Bonk \& C. Graham (dir.), Handbook of blended learning: global perspectives, local designs (p. 550-567). San Francisco, CA: Pfeiffer.

Clark, R. C. \& Kwinn, A. (2007). The new virtual classroom: evidence-based guidelines for synchronous elearning. San Francisco, CA : Pfeiffer éditeur, 
Depover, C., Karsenti, T. \& Komis, V. (2008). Enseigner avec les technologies. Québec, QC : Presses de l'Université du Québec.

Diaz, V. \& Brown, M. (2010). Blended learning. A report on the ELI focus session. Repéré à https:// docs.google.com/viewer? url=http\%3A\%2F\%2Fnet.educause.edu\%2Fir\%2Flibrary\%2Fpdf\%2FELI3023.pdf

Dziuban, C.D., Hartman, J.L. \& Moskal, P.D. (2004). Blended learning. Educause Center for Applied Research. Research Bulletin, 7, 1-12.

Ebersole, J.F. (2005). Meeting the quality challenge in online learning, Continuing Higher Education Review, 69, 69-76.

Frydenberg, J. (2007). Persistence in university continuing education online classes. International Review of Research in Open and Distance Learning, 8(3),1-15. Repéré à http://www.irrodl.org/ index.php/irrodl/article/view/375/934

Garrison, D.R. \& Kanuka, H. (2004). Blended learning: Uncovering its transformative potential in higher education. The Internet and Higher Education, 7, 95-105.

Garrison, D.R. \& Vaughan, N. (2008). Blended Learning in Higher Education: Framework, Principles, and Guidelines. San Francisco, CA: Jossey Bass.

Ginns, P. \& Ellis, R. (2007). Quality in blended learning: Exploring the relationships between online and face-to-face teaching and learning. The Internet and Higher Education, 10, 53-64.

Graham, C. R. (2006). Blended learning systems: Definition, current trends, and future directions. Dans C.J. Bonk \& C.R. Graham (dir.), The Handbook of blended learning: global perspectives, local designs (p. 8-26). San Francisco, CA: Pfeiffer.

Hwang, A. \& Arbaugh, J.B. (2009). Seeking feedback in blended learning: Competitive versus cooperative student attitudes and theirs links to learning outcome. Journal of Computer Assisted Learning, 25, 280-293.

Kelly, O. (2007). Moving to blended delivery in a Polytechnic: Shifting the mindset of faculty and institutions. Dans M. Bullen \& D.P. Janes (dir.), Making the Transition to E-Learning: Strategies and issues (p. 33-46). Hershey, PA: Information Science Publishing.

Khare, A. \& Lam, H. (2008). Assessing student achievement and progress with online examinations: Some pedagogical and technical issues. International Journal on E-Learning, 7(3), 383-402.

Kim, K-J., Bonk, C.J. \& Oh, E. (2008). The present and future state of blended learning in workplace learning settings in the United States. Performance Improvement, 47(8), 5-16.

Laster, S., Otte, G., Picciano, A.G. \& Sorg, S. (2005, avril). Redefining blended learning. Communication présentée au Sloan-C workshop on blended learning, Chicago, Illinois.

Leitner, H. (2008). Bit by bit: Innovating at the periphery to extend Harvard's core. Continuing Higher Education Review, 72, 163-183.

Lopez-Perez, M.V., Perez-Lopez, M.C., \& Rodriguez-Ariza, L. (2011). Blended learning in higher education: Students' perceptions and their relation to outcomes. Computers \& Education, 56, 818-826.

MacDonald, J. (2008). Blended learning and online tutoring: Planning learner support and activity design ( 2 éd.). Aldershot: Gower Publications. 
Masson, A., MacNeill, A., C. Murphy, C. \& Ross, V. (2008). The hybrid learning model - A framework for teaching and learning practice. iJET, 3, 12-17.

Morote, E-S., Wittmann, H. \& Kelly, T. (2007, mars). What really matters for both faculty to develop and implement hybrid/blended courses?Communication présentée à la conférence de la Society for information technology and teacher education international (SITE). San Antonio, Texas, ÉtatsUnis.

Noblet, J-P. (2011). L'appropriation d'un campus virtuel par les acteurs : une approche par la théorie de l'intéressement. Revue de l'Éducation à Distance, 25(3), 1-14.

Picciano, A.G. \& Dziuban, C.D. (dir.) (2007). Blended learning. Research perspectives. Needham, MA: Sloan Consortium.

Sandeen, C. (2008). Boomers, Xers, and Millenials: Who are they and what do they really want from continuing higher education? Continuing Higher Education Review, 72, 11-31.

Seok, S. (2008). Teaching aspects of e-learning. International Journal on E-Learning, 7(4),725-741.

Stacey, E. \& Gerbic, P. (2008, novembre et décembre). Success factors for blended learning. Communication présentée à la conférence annuelle de l'ASCILITE, Melbourne, Australie.

Thomas, H. (2007). An analysis of the environment and competitive dynamics of management education. Journal of Management Development, 26(1), 9-21.

Vaughan, N. (2007). Perspectives on blended learning in higher education. International Journal on E-Learning, 6(1), 81-94. Repéré à http://www.thefreelibrary.com/_/print/PrintArticle.aspx? $\mathrm{id}=159594390$

Wilson, G. (2007). New skills and ways of working : Faculty development for e-learning. Dans M. Bullen \& D.P. Janes (dir.), Making the Transition to E-Learning: Strategies and issues (p. 121-138). Hershey, PA : Information Science Publishing.

\section{NOTES}

1. Le Comité aviseur est composé de dix personnes nommées par le directeur du projet et qui représentent les enseignants, les étudiants et les directeurs des opérations impliqués ou non dans les technologies. Ce comité est consultatif.

\section{RÉSUMÉS}

Face à un environnement de plus en plus concurrentiel des écoles et facultés de gestion, l'apprentissage hybride devient une stratégie institutionnelle très prisée. Ce constat nous a permis de concevoir et d'implanter durant quatre sessions des années universitaires 2009 à 2011, un projet d'apprentissage hybride. Ce dispositif a été déployé dans dix cours distincts pour un total de 27 classes. Nous avons recueilli les perceptions des 805 étudiants inscrits dans ce projet pilote, notamment pour connaître leurs raisons de s'inscrire à un cours hybride, leurs appréciations des dispositifs pédagogiques hybrides ainsi que leurs points de vue des impacts sur 
les apprentissages. Les données colligées par questionnaire ont été analysées dans l'optique d'une évaluation de l'innovation pédagogique et de sa possible institutionnalisation. L'article se termine par une série de quatre propositions reliées à une conceptualisation émergente sur l'apprentissage hybride.

Dealing with an increasingly competitive environment, business schools and faculties are more and more engaged in hybrid learning approaches. This is why our institution designed and implemented a blended-learning project during the years 2009 to 2011 in ten distinct courses for a total of 27 groups. We gathered the perceptions of 805 students in order to better understand their reasons for enrolling in such a course, their level of satisfaction with the teaching and learning environment and their views regarding the impacts on learning. All the results coming from a questionnaire were analyzed in view of evaluating pedagogical innovation and its potential for institutionalization. The article ends with four proposals grounded in a theoretical framework on hybrid learning and teaching.

INDEX

Mots-clés : apprentissage en ligne, apprentissage hybride, évaluation, innovation pédagogique, sciences de gestion

\section{AUTEURS}

\section{JEAN-PIERRE BÉCHARD}

HEC Montréal

3000, chemin de la Côte-Sainte-Catherine

Montréal (Québec) H3T 2A7

jean-pierre.bechard@hec.ca

\section{CATHERINE CARRÉ}

Direction de l'apprentissage et de l'innovation pédagogique HEC Montréal

\section{GABRIELLA FRANKEL}

Direction de l'apprentissage et de l'innovation pédagogique HEC Montréal

\section{ZOFFIRATH DISSOU}

Direction de l'apprentissage et de l'innovation pédagogique HEC Montréal

\section{CÉLINE BAZINET}

Direction des programmes de certificat HEC Montréal

\section{GILLES CAZABON}

Management

HEC Montréal 


\section{YVES GOYETTE}

Service de l'audiovisuel

HEC Montréal 